\title{
Noninvasive assessment of pressure drop in mitral stenosis by Doppler ultrasound
}

\author{
L. HATLE, A. BRUBAKK, A. TROMSDAL, AND B. ANGELSEN \\ From Section of Cardiology, Medical Department, University Hospital, 7000 Trondheim; and Division of \\ Engineering Cybernetics, The Norwegian Institute of Technology and Division of Automatic Control at the \\ Foundation of Scientific and Industrial Control at the University of Trondheim, 7000 Trondheim, Norway
}

SUMMARY A noninvasive method is described for measuring the pressure drop across the mitral valve in mitral stenosis by Doppler ultrasound. A maximum frequency estimator was used to record maximum velocity in the Doppler signal from the mitral jet. Provided the angle between the ultrasound beam and the maximum velocity is close to zero the pressure drop can be calculated directly.

Good correlation was found between Doppler measurements and simultaneous pressure recordings during heart catheterisation in 10 patients. No false negative or false positive diagnoses of mitral stenosis were made among 55 patients ( 35 patients with mitral stenosis and 20 patients with other valve lesions). The measurements were easy to perform in most patients and the method seems well suited both to diagnose and to follow patients with mitral stenosis.

Holen et al. (1976) have shown that the pressure drop across a stenotic mitral valve may be calculated from transcutaneous Doppler ultrasonic measurements of the blood velocity in the mitral jet. A pressure difference across the valve is necessary both to accelerate the blood through the valve constriction and to overcome the viscous resistance. The work of Holen et al. indicates that viscous resistance may be neglected for the blood with the highest velocity in the jet. The pressure drop across the valve may then be calculated from the maximum velocity in the jet using the Bernoulli equation.

The ultrasound reflected from a small blood sample will undergo a change in frequency (Doppler effect) which is proportional to the velocity of the blood and to the cosine of the angle between this velocity and the ultrasonic beam. This angle can be approximated to zero for measurements of mitral jet velocity from the chest. The maximum velocity in the jet can, therefore, be calculated from the maximum frequency shift in the Doppler signal. Holen et al. used a complete spectral analysis to obtain the maximum frequency shift in the Doppler signal. In the present study a simple maximum frequency estimator is used (Brubakk et al., 1977), and the time variation of the frequency shift can be recorded on an ordinary paper recorder.

Received for publication 4 May 1977
The purpose of the present study was to evaluate the clinical usefulness of the maximum frequency estimator for assessment of the pressure drop in mitral stenosis. Ultrasonic measurements were done simultaneously with pressure recordings during heart catheterisation in 10 patients with mitral stenosis.

\section{Methods}

THEORETICAL CONSIDERATIONS

A constriction in a tube accelerates fluid which passes through it. This acceleration is called convective, since it is caused by the convection of the fluid from one point in space with one velocity to another point in space with a different velocity. Convective acceleration occurs in flow through a stenotic mitral valve as blood passes from comparatively low velocity in the left atrium $(0.2 \mathrm{~m} / \mathrm{s})$ to higher velocity $(\sim 2 \mathrm{~m} / \mathrm{s})$ in the jet. Additional acceleration is caused by changes in blood flow velocity during diastole, especially at the time of mitral valve opening and closure.

Acceleration of a mass requires a force. A small fluid element of volume $\Delta \mathrm{V}$ may be regarded as a body with mass $\rho \cdot \Delta \mathrm{V}$, where $\rho$ is the density $\left(1.06 \times 10^{3} \mathrm{~kg} / \mathrm{m}^{3}\right.$ for blood) of the fluid per unit volume. Acceleration of this element through a stenotic valve is achieved by the pressure drop 


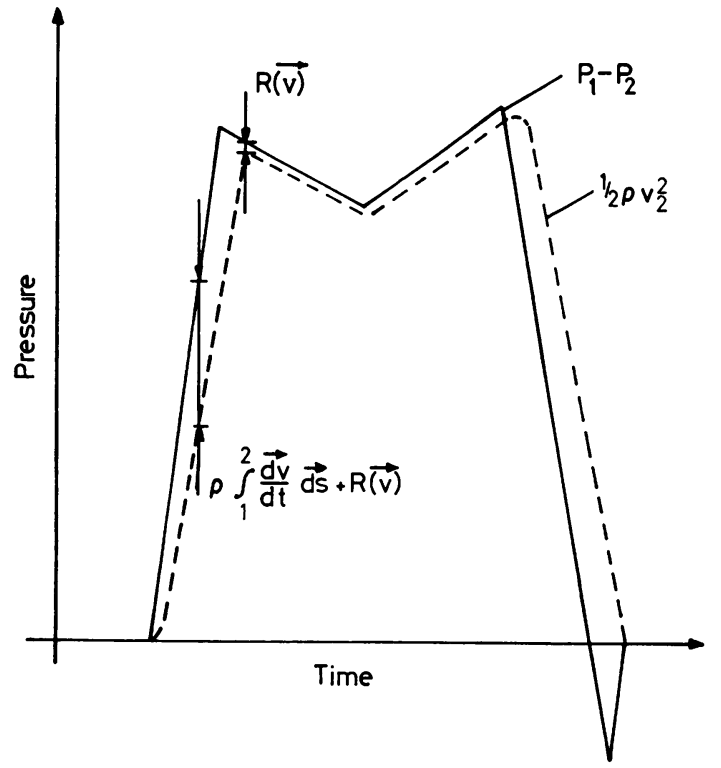

Fig. 1 Contribution of the different terms in Eq. (1) to the total pressure drop across a stenotic valve. The relative values of the terms are arbitrarily chosen.

across the valve. The relation between the pressure drop and the velocity is given by the Bernoulli equation with an added viscous term

$\mathbf{P}_{1}-\mathbf{P}_{2}=\frac{1}{2} \rho\left(\mathrm{v}_{2}{ }^{2}-\mathrm{v}_{1}{ }^{2}\right)+\rho \int_{1}^{2} \frac{\overrightarrow{d v}}{\mathrm{dt}} \overrightarrow{\mathrm{ds}}+\mathbf{R}(\overrightarrow{\mathrm{v}})$

where suffix 1 denotes the position of the fluid element in front of the valve, and suffix 2 in the valve jet; $P$ is the pressure, $\vec{v}$ is the velocity vector of the fluid element along its path, and $\overrightarrow{d s}$ is the path element.

The first term of the right-hand side of the equation represents the convective acceleration, the second term represents the acceleration caused by changes of the velocity with time, while the last term represents viscous losses.

Estimates of the magnitude of the first and second terms may be given. The atrial velocity, $v_{1}$, is $\sim 0.2 \mathrm{~m} / \mathrm{s}$. The value of the jet velocity $\mathrm{v}_{2}$ will depend on the degree of stenosis, usually being $1-3 \mathrm{~m} / \mathrm{s}$. We thus see that $\mathrm{v}_{2}{ }^{2} \gg \mathrm{v}_{1}{ }^{2}$ so that $\mathrm{v}_{1}$ may be neglected in the convective acceleration term in Eq. (1). For $v_{2}=2 \mathrm{~m} / \mathrm{s}$, the convective term represents a pressure drop of $16 \mathrm{mmHg}$ (Fig. 1).

For the second term we assume that the velocity increases from a zero value in the atrium to the maximum jet velocity $\mathrm{v}_{2}$ proportionally to the square of the distance. The time dependency of the velocity is assumed to be the same along the whole path of the fluid. This term then takes the form

$\frac{\rho l}{3} \cdot \frac{\mathrm{dv}_{2}}{\mathrm{dt}}$

where $l$ is the path length of the fluid element from the atrium to the jet. For a stenosis with maximum velocity of $2 \mathrm{~m} / \mathrm{s}$, typical values at the valve opening and closure are (see Figs. 2 and 3).

$$
\begin{aligned}
& \frac{\mathrm{dv}_{2}}{\mathrm{dt}}=30 \text { to } 40 \mathrm{~m} / \mathrm{s}^{2} \\
& \quad l=5 \cdot 10^{-2} \mathrm{~m} \\
& \frac{\rho l}{3} \cdot \frac{\mathrm{dv}_{2}}{\mathrm{dt}}=4 \text { to } 5 \mathrm{mmHg}
\end{aligned}
$$

We thus see that this term is of the same order as the convective pressure drop during valve opening and closure. When the valve is open, however, $\mathrm{dv}_{2} / \mathrm{dt} \sim 1 \mathrm{~m} / \mathrm{s}$, which gives

$\frac{\rho l}{3} \cdot \frac{\mathrm{dv}_{2}}{\mathrm{dt}} \sim 0.2 \mathrm{mmHg}$

which implies that the convective term dominates the acceleration during diastole.

The magnitude of the viscous losses is difficult to estimate. The viscous losses arise from friction between the fluid element and its neighbouring fluid, and will thus not only depend on $v_{2}$, but on the whole velocity profile. However, our findings together with those of Holen et al. suggest that for the fluid element with the maximum velocity in the mitral jet, the viscous losses may be neglected.

In Fig. 1 the contribution of the different terms to the total pressure drop is seen. The relative values of the terms are arbitrarily chosen. Because of the second acceleration term in Eq. (1) there is a delay between the pressure drop and $\frac{1}{2} \rho \mathrm{v}_{2}{ }^{2}$. In addition this term requires a negative pressure drop to arrest the blood flow at the end of the diastole. When $\mathrm{dv}_{2} / \mathrm{dt}=0$, the second term is zero, and the difference between the pressure drop and $\frac{1}{2} \rho v_{2}{ }^{2}$ is given by viscous losses only.

By neglecting the second acceleration term and the viscous losses, the pressure drop can be calculated from $v_{2}$ alone. Inserting the value for $\rho$ in Eq. (1), the following simple formula is found

$\mathrm{P}_{1}-\mathrm{P}_{2}=4 \cdot \mathrm{v}_{2}{ }^{2}$ ( $\mathrm{mmHg}$ )

where $v_{2}$ is in units of $\mathrm{m} / \mathrm{s}$, while the pressure drop is found in units of $\mathrm{mmHg} . P_{1}$ is the atrial pressure, and $P_{2}$ is the pressure in the jet where $v_{2}$ is achieved. $\mathrm{P}_{\mathbf{2}}$ will actually be less than the ventricular pressure since a pressure drop working against the flow is needed to retard the large jet velocity to a much smaller ventricular velocity. However, most of the 

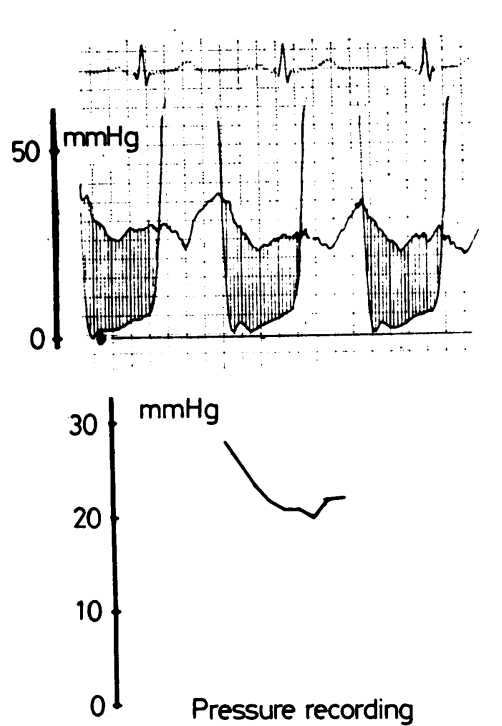
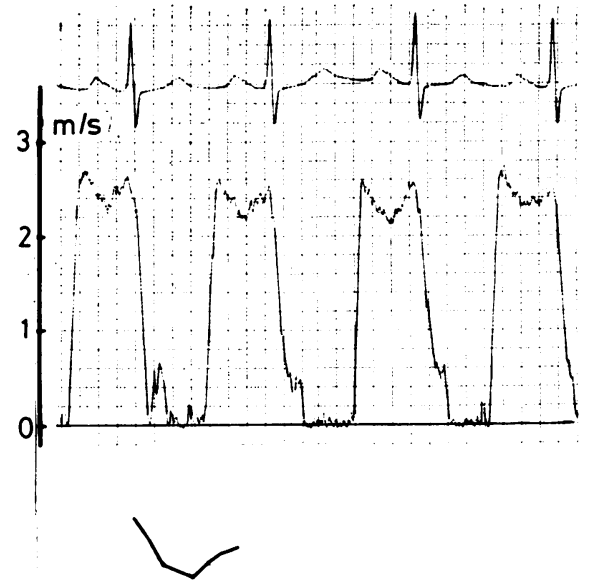

Ultrasonic Doppler recording
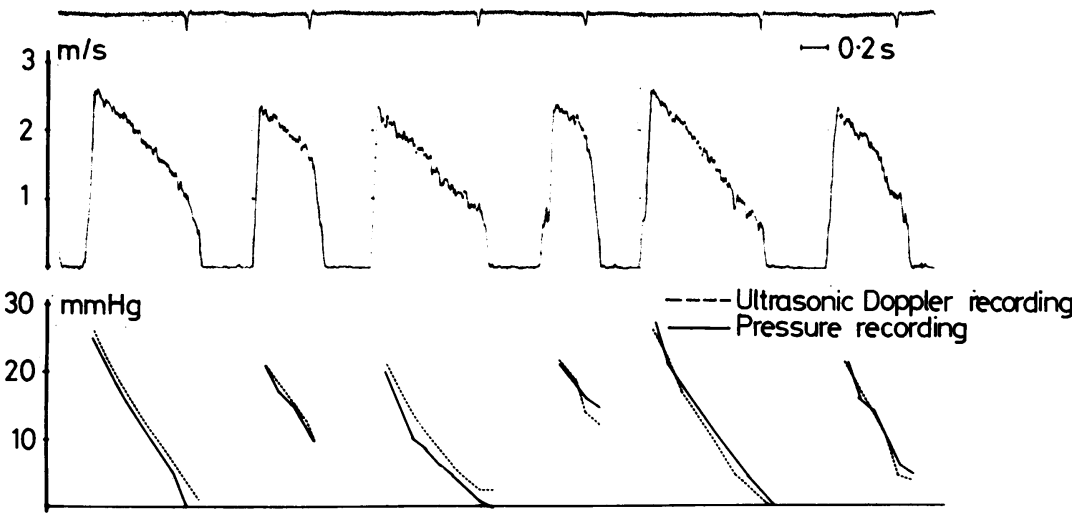

Fig. 3 Case 4. Simultaneous ultrasonic (above) and pressure recording (below) with calculated and measured pressure drop during diastole (middle).

Fig. 2 Case 8. Above left: Left ventricular and pulmonary capillary venous pressure. Above right: Ultrasonic recording. Below: Measured and calculated pressure drop during diastole.

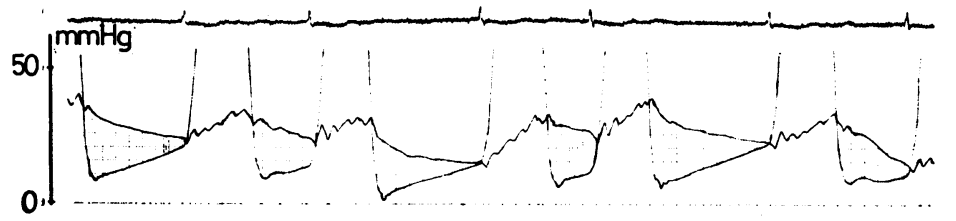

kinetic energy in the jet is lost in turbulence and post-valvular vortices, so that this pressure drop is probably not large.

The maximum velocity in the jet, $\mathrm{v}_{2}$, is calculated from the maximum frequency shift, $f_{2}$, of the Doppler signal according to the following equation $\mathrm{v}_{2}=\frac{\mathrm{c}}{2 \mathrm{f}_{0}} \cdot \frac{\mathrm{f}_{2}}{\cos \phi}$ $f_{0}$ is the transmitted ultrasonic frequency, $c$ is the velocity of sound in the blood, and $\phi$ is the angle between the ultrasonic beam and the direction of the maximum velocity. By proper angling from the chest, a small value of $\phi$ can be obtained so that $\cos \phi=1$ is a good approximation. This approximation is used in the work, thereby enabling us to estimate $v_{2}$ from $f_{2}$. 
Table

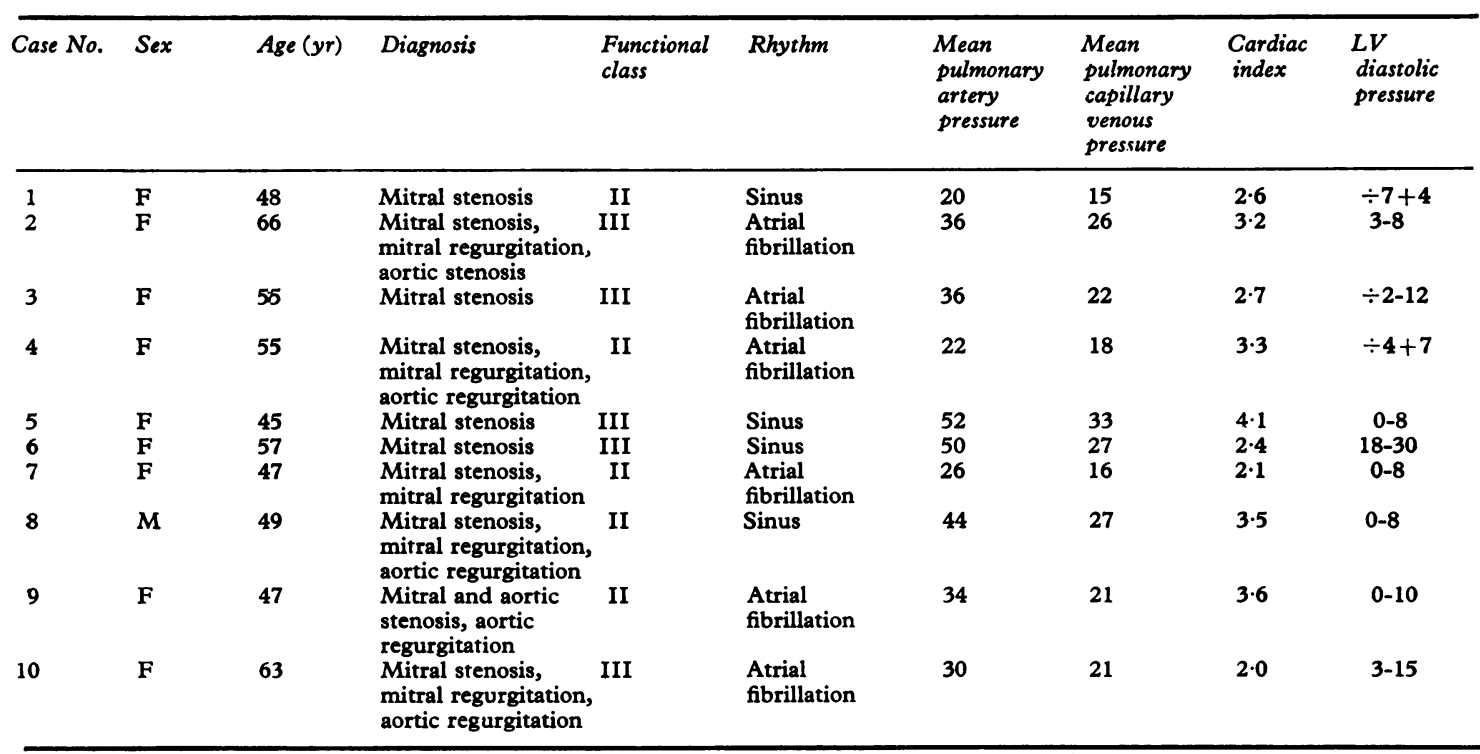

\section{CLINICAL PROCEDURE}

In 10 patients with mitral stenosis simultaneous ultrasonic and pressure recordings were performed during evaluation for surgery. There were 9 women and 1 man, aged 45 to 66 years: 5 patients had pure mitral stenosis, and 5 had combined mitral stenosis and regurgitation; 2 had additional aortic stenosis and $\mathbf{4}$ had aortic regurgitation. Four patients were in sinus rhythm, 6 had atrial fibrillation. In 4 of the patients mitral commissurotomy had been done several years previously. Functional class, heart rhythm, and catheterisation data are given in the Table. Heart size varied from 450 to $870 \mathrm{ml} / \mathrm{m}^{2}$. Ultrasonic recordings were performed before or after heart catheterisation in 25 other patients with mitral stenosis and in 20 patients with other valve lesion, but without mitral stenosis.

Right and left heart catheterisation was done percutaneously from a cubital vein and the femoral artery, respectively. Pressure was measured with an Elema Schoenander transducer, type EMT 35, and recorded on a Mingograph 81. Zero level for pressure measurements was the anterior axillary line in the 4th intercostal space. Cardiac output was determined by the Fick method. Pressures were recorded simultaneously in the pulmonary wedged position (PCV pressure) and the left ventricle, both before and immediately after left ventricular angiography. The mitral valve pressure drop was measured as the difference between the PCV pressure advanced $0.06 \mathrm{~s}$ and the left ventricular pressure.
The ultrasonic Doppler instrument used for measuring mitral flow velocity is described in detail elsewhere (Angelsen, 1975, 1976). The instrument can be used either in a pulsed mode or as a continuous wave meter. The ultrasonic frequency is $\frac{\mathrm{D}}{\mathrm{d}}$ $2 \mathrm{MHz}$. In the pulsed mode, using repetition frequencies of 6.7 and $9.8 \mathrm{KHz}$, velocities up to $1.7 \mathrm{~m} / \mathrm{s}$ can be measured within $7 \mathrm{~cm}$ from the transducer and up to $1 \mathrm{~m} / \mathrm{s}$ within $12 \mathrm{~cm}$. The velocities were measured in a cylindrical volume of $15 \mathrm{~mm}$ diameter and $7.5 \mathrm{~mm}$ length. In the continuous mode, velocities up to $5 \mathrm{~m} / \mathrm{s}$ can be measured but with loss of range resolution.

The transducer was placed over or slightly medial to the apex of the heart and angled until the characteristic sound of mitral flow was detected. The audible Doppler signal in mitral stenosis is a harsh sound containing high frequencies from the $\rightarrow$ large jet velocity and differs clearly from the sound obtained from normal blood flow. The direction $N$ of the transducer was carefully adjusted until the maximum Doppler shift was found. The maximum and mean velocities were recorded together with the electrocardiogram. From the maximum velocity, the pressure drop between the left atrium and the left ventricle was calculated for each $0.1 \mathrm{~s}$ during $\mathscr{D}$ diastole, using Eq. (5). The calculation was per-? formed both for single beats and as an average for several consecutive beats and compared with $\stackrel{\vec{D}}{\mathrm{D}}$ simultaneous pressure recordings. The mean $\frac{?}{8}$ pressure drop during diastole was obtained from $\varrho$ the average diastolic curves of 10 to 30 beats? 
recorded simultaneously with pressure transducer and ultrasound.

Ultrasonic and pressure recordings were made both before and after left ventricular angiography in 2 patients also with raised legs to increase venous return. In addition, ultrasonic recordings were repeated within minutes several times the day before and one or two days after catheterisation to test the reproducibility of the method.

\section{Results}

Fig. 2 (right) shows the maximum velocity curve measured by ultrasound in case 8 . This curve is typical for severe mitral stenosis in a patient with sinus rhythm. After the opening of the mitral valve, there is a rapid increase in blood flow velocity from zero to its maximum value. After the initial peak, there is a slight decrease until the second peak appears after atrial contraction. Below the velocity curve the derived pressure drop is shown, while to the left the results from the simultaneous pressure measurements are seen. The pressure curve obtained from the velocity is similar to that obtained by catheterisation. The mean diastolic pressure drop was $23 \mathrm{mmHg}$ with both methods. In severe mitral stenosis the pressure drop remains high throughout diastole, which is also found for the velocity curve. Peak velocity in this case was $2.8 \mathrm{~m} / \mathrm{s}$ and the velocity at the end of diastole was only slightly lower.
In atrial fibrillation (Fig. 3) a different curve is obtained. The initial peak is followed by a gradual decrease in velocity, and the second peak seen in patients with sinus rhythm is absent. The peak velocity varies more than in patients with sinus rhythm as a short diastole in one or more of the preceding beats results in a higher peak velocity in the following beat. The variation in mean pressure drop from beat to beat is easily seen from the ultrasonic as well as from the pressure recording. The figure also shows how the pressure drop calculated from the ultrasonic recording during diastole for each beat is very similar to that obtained by catheterisation. Adequate correlation can also be obtained for a longer time, as shown in Fig. 4.

These examples show close agreement between pressure drops calculated from ultrasonic and catheterisation recordings when good ultrasonic signals were obtained. With a recording of poorer quality spuriously low maximum velocities will be found. Fig. 5 shows examples of some of the problems associated with this method. In curve a, the initial peak velocity is less than expected when compared with simultaneous pressure recordings. However, this deficiency can be observed from the ultrasonic recording alone as the initial peak velocity should not be less than the atrial peak. The curve also shows the false results which may occur when the velocity exceed $1.7 \mathrm{~m} / \mathrm{s}$ for the pulsed mode of the instrument. Because of incomplete filtering in the instrument a falsely high velocity is observed.
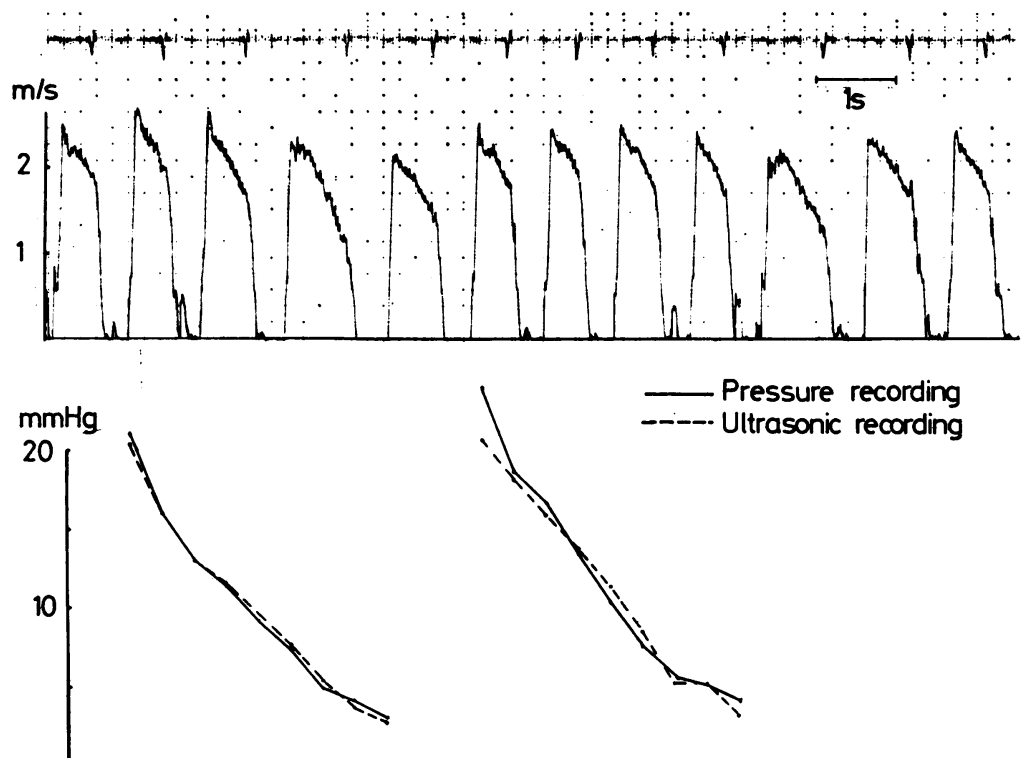

Fig. 4 Case 4. Above: Ultrasonic recording. Below: Calculated pressure dropcompared with simultaneous pressure recording. (a) Before $L V$ angiography-mean of 44 consecutive beats; (b) after $L V$ angiography-mean of 50 consecutive beats. 
The next curve, $b$, shows the comparison between simultaneous ultrasonic and pressure recordings when the continuous mode was used and beats showing loss of initial peak velocity were omitted. In this patient good Doppler signals throughout diastole were difficult to obtain for more than a few beats at a time, perhaps because of hyperventilation. Both methods (curve $\mathrm{C}$ ) show increase in pressure drop after the patient's legs had been elevated but, as can be seen from the ultrasonic recording, some of the initial peak velocity is still lost. The difference in mean diastolic pressure drop is $3.5 \mathrm{mmHg}$.

After left ventricular angiography a better ultrasonic recording was obtained (d), and then the calculated pressure drop was similar to the simultaneous pressure recording $(16-17.5 \mathrm{mmHg})$.

Fig. 6 shows part of the recordings from a patient with atrial fibrillation. In the first and third beat, a difference is noted between pressure and ultrasonic recordings, while in the following beats the difference is very small. A recording showing many of the former beats would underestimate the mean pressure drop. From the velocity curve, however, it can be seen that the ultrasonic recording of the first and third beats differs from the rest. In particular the first beat shows a more rapid decline in velocity.
Fig. 7 shows the least satisfactory of the ultrasonic curves recorded. The pressure drop calculated from the velocity curves is underestimated for most beats. The difference in mean diastolic pressure drop with the two methods (calculated from 30 beats) was $4 \mathrm{mmHg}$. However, calculating the pressure drop from only the 'best' beats recorded gave a result closer to the pressure drop obtained by catheterisation.

The mean and peak diastolic pressure drop for the 10 patients with simultaneous pressure and ultrasonic recordings before and after left ventricular angiography are shown in Figs. 8 and 9. The correlation is good except for two patients. The enclosed symbols marked represent a patient in whom, after left ventricular angiography, the pulmonary ज arterial instead of pulmonary capillary venous pres- $N$ sure was used to obtain the pressure drop. This pressure drop was consistently about $5 \mathrm{mmHg}$ higher than that obtained from the velocity curves. Before angiography when pulmonary capillary venous pressure was measured the two methods gave nearly identical results.

The other patient represented by a square was the only one for whom pressure drop calculated from velocity was higher than that calculated from catheterisation data. However, raised left ventricular diastolic pressure was found in this patient without 

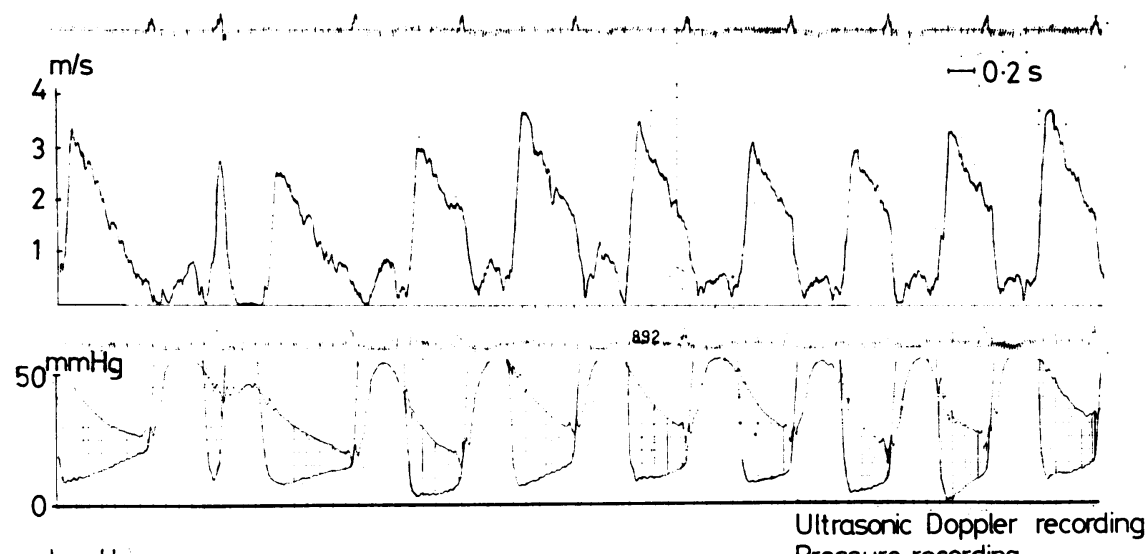

Fig. 6 Case 3. Above:

Ultrasonic recording. Middle: Left ventricular and pulmonary capillary pressure.

Below: Pressure drop during diastole - calculated and measured from the above curves.

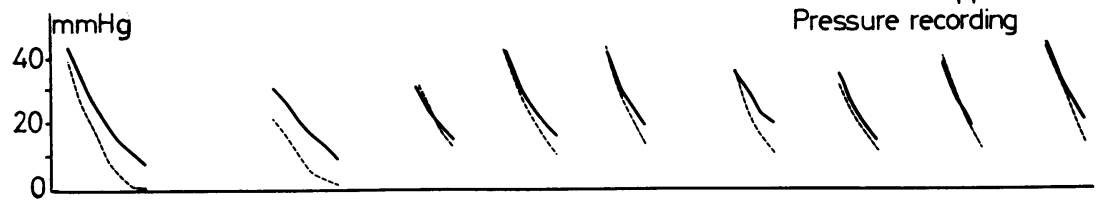

satisfactory explanation. Left ventricular angiography showed normal volumes and contractions with minimal mitral regurgitation. Moderate systemic hypertension and electrocardiographic signs of left ventricular hypertrophy were present, but the left ventricular pressure curve was equally raised throughout diastole without a significant end-diastolic increase or a conspicuous a-wave. The ultrasonic record, on the other hand, was of good quality and several recordings gave similar results. Though the zero level of the pressure transducer was routinely checked both before, during, and after catheterisation, the possibility of erroneous zero line cannot be excluded.

Fig. 10 shows the variation in pressure drop, measured by ultrasound, in one patient. Curve a shows the diastolic pressure drop measured the day before catheterisation. The unbroken line is the pressure drop at rest, while the stippled line shows the increase in pressure drop obtained by raising the patient's legs to increase venous return. Curve $b$, with a larger pressure drop, was recorded in the catheterisation room before the start of the procedure. Continuous ultrasonic recordings (curve 


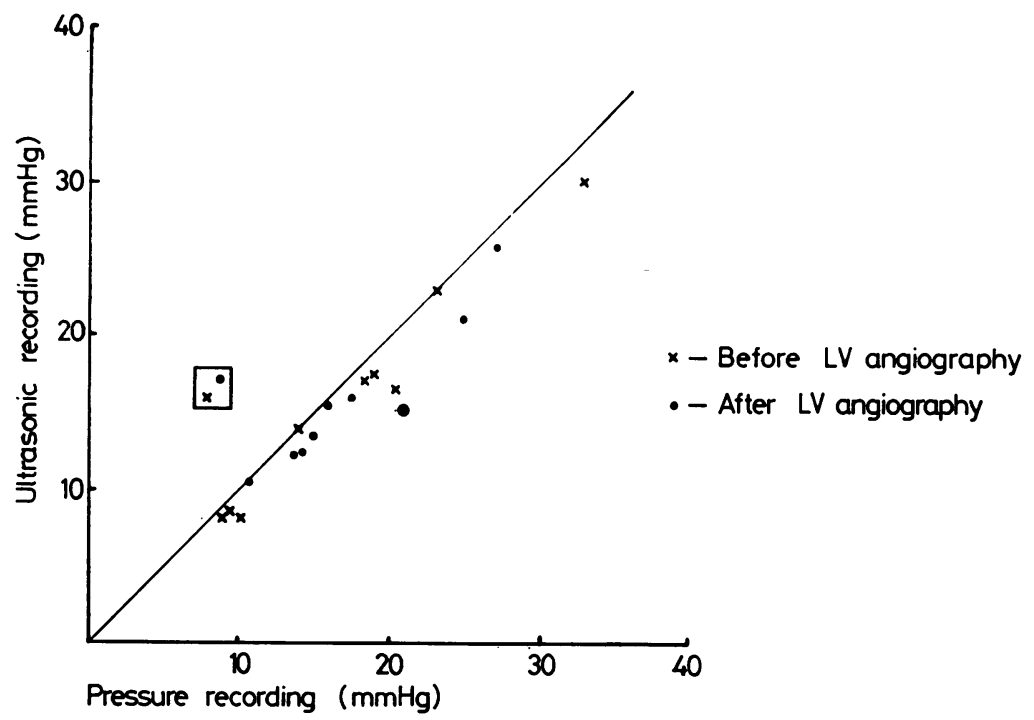

Fig. 8 Mean diastolic pressure drop in 10 patients.

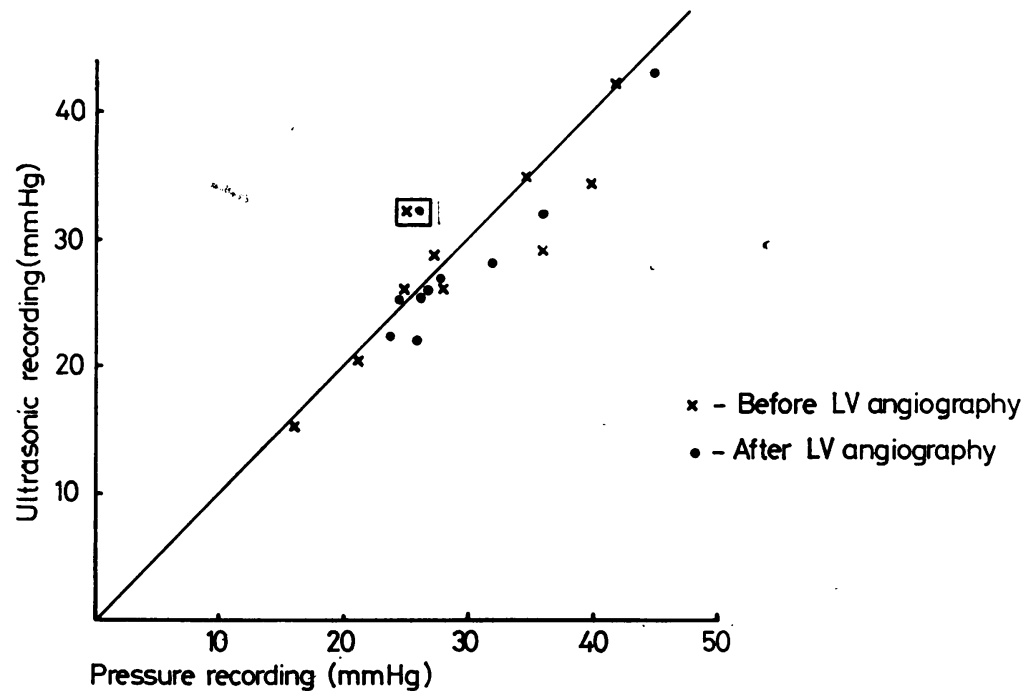

Fig. 9 Peak diastolic pressure drop in 10 patients.

c) showed an abrupt increase in peak velocity immediately the catheterisation procedure began with venous puncture. During catheterisation several recordings (curve d) showed similar results, except after left ventricular angiography (curve e) when a further increase in pressure drop was noted. The last curve ( $f$ ) obtained the next day is similar to the first one. The increase in pressure drop after left ventricular angiography was confirmed by simultaneous pressure recordings.

Fig. 11 shows the results of repeat ultrasonic recordings in 9 of the patients studied. In most patients, the average pressure drop was higher $N$ during catheterisation than on the previous or $\mathrm{CU}^{\mathrm{N}}$ following day, and was further increased after angiography. In two patients the lower pressure drop after angiography was associated with a slower $\frac{}{\odot}$ heart rate. Repeat measurements on a particular $\stackrel{\oplus}{?}$ day showed only small variations.

In the 25 patients with mitral stenosis, on whom catheterisation and ultrasonic recordings were $\mathbb{\Omega}^{\mathbb{D}}$ performed on different days, the velocity curves $\mathbb{\mathbb { D }}$ gave pressure drops within 20 per cent of the ones calculated from the pressure curves, except in one 

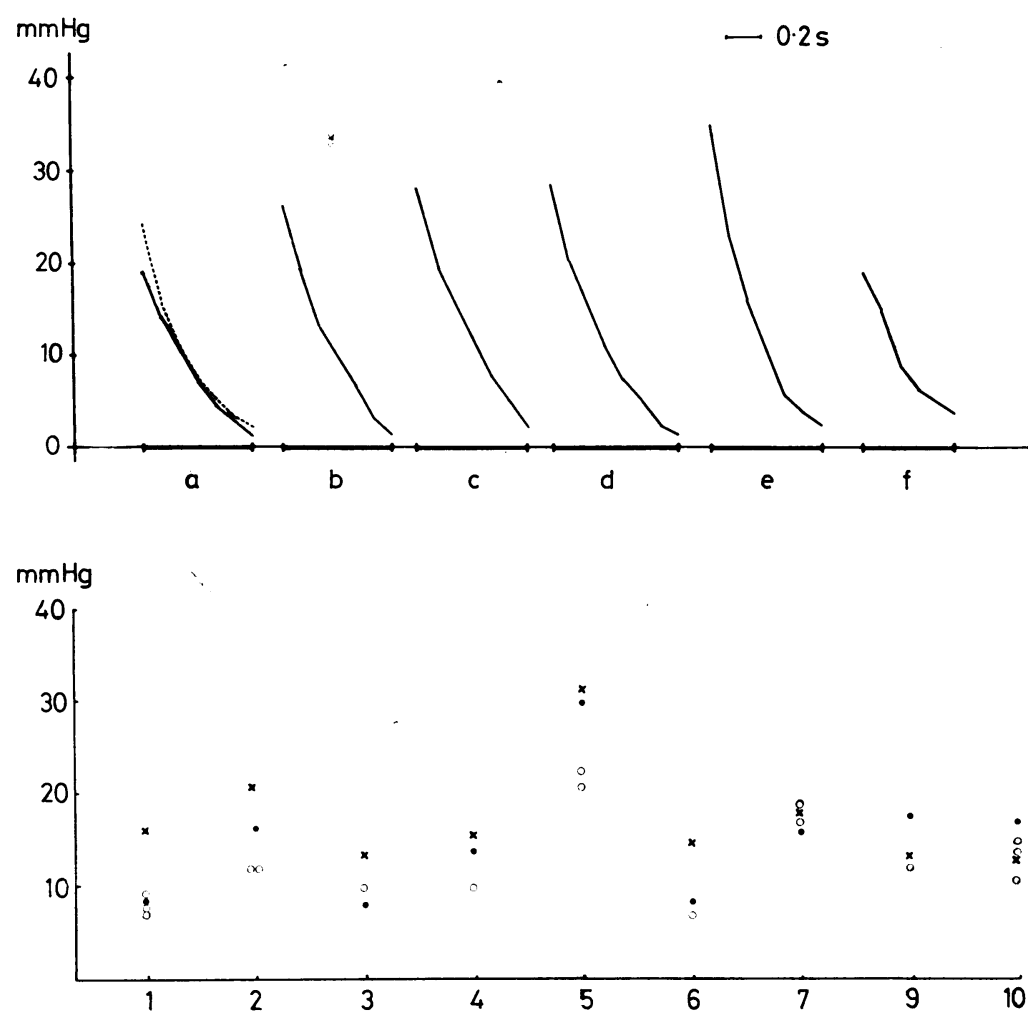

Fig. 10 Pressure drop during diastole-repeat ultrasonic recordings in case 2. (a) Day before catheterisation, stippled curve shows pressure drop with raised legs. ( $b, c, d$, and $e$ ) In the catheterisation room, $(e)$-after $L V$ angiography. $(f)$ Day after catheterisation. In all curves the pressure drop is calculated from a mean of 20 to 50 consecutive beats.
Fig. 11 Mean diastolic pressure drop in 9 patients. $\bigcirc$ At rest, on different days; $\bigcirc$ during catheterisation; $\times$ after $L V$ angiography. patient where ultrasonic measurements were unsatisfactory.

In the patients with other valve lesions, but without mitral stenosis, ultrasonic recordings indicated a pressure drop from the left atrium to the left ventricle of 2 to $4 \mathrm{mmHg}$ early in diastole decreasing to zero in mid-diastole. In patients with sinus rhythm a late diastolic increase to 1 to $2 \mathrm{mmHg}$ was seen after atrial contraction. In patients with more than moderate mitral regurgitation the initial pressure drop was higher, 6 to $8 \mathrm{mmHg}$, but with rapid decline giving a mean pressure drop less than 3 to $4 \mathrm{mmHg}$.

\section{Discussion}

The present method depends upon the assumption of a zero angle between the ultrasonic beam and the maximum jet velocity, and the validity of neglecting viscous losses. A small viscous pressure drop may be masked by a reverse deceleration pressure drop between the ventricle and the mitral jet (see theoretical considerations). This reverse pressure drop is probably small, which is confirmed by Yellin and Peskin (1975) in their in vitro study of pressure flow relations across a tube constriction.
The adequate correlation between pressure drop estimated by ultrasonic recording using Eq. (5) and that obtained by catheterisation, as shown in Figs. 2, 3, and 4, confirm that viscous losses are negligible.

In the patients for whom the pressure drop was underestimated from ultrasonic measurements, viscous losses were unlikely to have been the cause of the error, as differences between the two methods varied from beat to beat (Figs. 5 and 6). In these cases improper angling of the ultrasonic beam is more likely to have been responsible. Several transducer positions may have to be tried before the maximum velocity is found. If the angle between the ultrasonic beam and the maximum jet velocity differs substantially from zero, the cosine of the angle will be less than 1 and the maximum velocity and the pressure drop will be underestimated (Eq. 6).

In Fig. 7 the pressure drop measured by ultrasound is about 20 per cent lower than that obtained during heart catheterisation, which could be caused by an angle $\phi=26^{\circ}$. A smaller angle than this should be obtained in most patients, as the jet is divergent and the maximum velocity may therefore be recorded from more than one position. The underestimate 
of the pressure drop of about 20 per cent in this patient was also the largest in this study. When heart catheterisation and ultrasonic recordings were performed on different days in 25 additional patients with mitral stenosis, the underestimate was also within 20 per cent with one exception. A satisfactory position for recording maximum velocity was found in 34 of the 35 patients examined. In the exceptional case optimal audio signal and velocity curves could not be obtained. The reason for this failure may be anatomical, such as emphysema, rotation of the heart, or a mitral valve deformity causing the jet to be directed away from the area on the chest wall from where it can be recorded. In all other patients the results were easily repeated on several occasions and by different observers.

Since the possibility of underestimating the pressure drop exists, it is important to know whether this can be recognised from the ultrasonic recordings. An analysis of a large number of beats recorded simultaneously with the two methods in the 10 patients indicates that significant underestimates could usually be recognised from the velocity curve. In sinus rhythm the deviations in curve form were easily seen, as shown in Fig. 5. Furthermore, in sinus rhythm beat-to-beat variations were usually small. Greater variations were observed in one patient only-probably because of increased respiration. In atrial fibrillation variations in peak velocity with varying heart rate gave similar results to the simultaneous pressure recording. However, when a varying decline in velocity during diastole was noted (Fig. 6), maximum velocity/pressure drop was underestimated in the beats with the more rapid decline. The results indicate that a significant underestimate is not likely to occur if care is taken to obtain a satisfactory ultrasonic recording.

In all patients with mitral stenosis the diagnosis was confirmed by finding abnormally high velocities. A false positive diagnosis may be made in patients with severe aortic regurgitation in whom a rapid flow towards the transducer during diastole may be recorded from the chest. In patients with atrial fibrillation the velocity curve may resemble that obtained in mitral stenosis. In sinus rhythm, differentiation is easier-as the increase in mitral velocity after atrial contraction is not seen. By changing the direction of the ultrasonic beam the velocity in the mitral and aortic valve areas may be differentiated (Brubakk et al., 1977).

Flow through the regurgitant aortic valve also starts immediately after the aortic component of the second heart sound. A false positive diagnosis of mitral stenosis was not made among 4 patients with significant aortic regurgitation.

Our results confirm that the maximum velocity $\stackrel{\overrightarrow{\vec{F}}}{\rightarrow}$ estimator gives correct values both at high and low velocities. This was especially well seen in patients with atrial fibrillation and slow ventricular rate $\frac{\bar{c}}{7}$ where an initial high velocity was gradually reduced $\stackrel{\varnothing}{\varrho}$ to nearly zero, indicating a pressure drop less than $1 \mathrm{mmHg}$ at the end of diastole. In contrast to a complete spectral analysis, the maximum frequency estimator gives an output in the form of analogue $\vec{\omega}$ voltages, which may be displayed instantly on an ordinary paper recorder.

The present data indicate that the pressure drop 0 across a stenotic mitral valve can be measured with î acceptable accuracy using transcutaneous Doppler $\vec{\omega}$ ultrasound. Additional information can be obtained by ultrasonic recording during exercise, and the 을 method can be used in noninvasive diagnosis, $\vec{T}$ assessment, and follow-up of patients with mitral of stenosis.

\section{Addendum}

Since the preparation of this paper we have made simultaneous measurements in 10 more patients where the pressure drop across the mitral valve ranged from 4 to $28 \mathrm{mmHg}$. In these patients even better correlation between ultrasound and pressure recordings was found, probably because of more $\varrho$ experience with the ultrasound method.

\section{References}

Angelsen, B. A. J. (1975). Transcutaneous Measurement of Aortic Blood Velocity by Ultrasound. A Theoretical and $\vec{\Phi}$ Experimental Approach. Report 75-78-W, Division of Engineering Cybernetics, Norwegian Institute of Tech-? nology, University of Trondheim, Norway.

Angelsen, B. A. J. (1976). Analcg Estimation of the Maximum Frequency of Doppler Spectra in Ultrasonic Blood Velocity 0 Measurements. Report 76-21-W, Division of Engineering Cybernetics, Norwegian Institute of Technology, University $\mathrm{O}$ of Trondheim, Norway.

Brubakk, A. O., Angelsen, B. A. J., and Hatle, L. (1977). D Diagnosis of valvular heart disease using transcutaneous Doppler ultrasound. Cardiovascular Research, 11, 461- ज
469.

Holen, J., Aaslid, R., Landmark, K., and Simonsen, S. N (1976). Determination of pressure gradient in mitral 0 stenosis with a non-invasive ultrasound Doppler technique. Acta Medica Scandinavica, 199, 455-460.

Yellin, E. L., and Peskin, C. S. (1975). Large amplitude pulsatile water flow across an orifice. Transactions of the ASME. Series G. Fournal of Dynamic Systems, Measurement, \& and Control, 97, 92-95.

Requests for reprints to Dr Liv Hatle, Section of $\frac{0}{\stackrel{0}{O}}$ Cardiology, University Hospital, 7000 Trondheim, Norway. 